\title{
COVID-19 vaccine hesitancy is positively associated with affective wellbeing
}

\author{
Mayank Kejriwal $^{\mathrm{a}, 1,2}$ and Ke Shen ${ }^{\mathrm{a}, 1}$

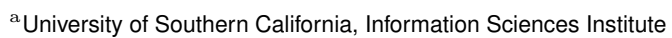

This manuscript was compiled on March 7, 2021

\begin{abstract}
SARS-CoV-2 (COVID-19) vaccine hesitancy in the United States is currently at a high level. To enable a better understanding of this hesitancy, this study explores the association between affective wellbeing measures and COVID-19 vaccine hesitancy. We consistently find a positive association between the two, regardless of which of ten different affect state variables (two positive, and eight negative) or their composite, is used. For example, people who experience more worry or anxiety (two negative affect wellbeing states) are less vaccine-hesitant, and vice versa. The association is found to hold even when controlling for potential confounds such as income bracket, political affiliation, gender and employment status. Associations are strongest for the fully employed, and for those in the middle-class income bracket. While consistent at the national level, considerable dispersion is found at the county level. We discuss the implications of these findings briefly.
\end{abstract}

Vaccine Hesitancy | Affective Wellbeing | COVID-19

$\mathbf{R}$ ecent news and data from polls have shed an alarming light on vaccine hesitancy and its costs, e.g. The New York Times recently referred to it as vaccine alarmism (1), vaccine hesitancy has been cited as the next challenge in the fight against COVID-19 (2), and even healthcare workers are hesitant about getting vaccinated (3). Even before COVID19, vaccine hesitancy and (consequent) under-vaccination of target populations (including children) had been an area of concern for health policy (4-6). While this brief report does not attempt to uncover causal drivers of COVID-19 vaccine hesitancy, it aims to understand whether affective wellbeing is a potential correlate of vaccine hesitancy, and more importantly, whether any such results robustly hold for multiple measures of affective wellbeing in the face of controls that include socio-demographic variables, employment status and political party affiliation. Understanding such affective correlates may help devise better policies in the near future for mitigating hesitancy.

This study is based on an analysis of recent survey data collected by Gallup. An important aspect of Gallup's surveys is measurement of subjective wellbeing (SWB) (7-9). In the literature, SWB has been measured in a variety of ways, but two that have extensively used are life satisfaction $(10,11)$ and affective wellbeing $(12,13)$. We consider the latter in this study, as it is asked at a finer granularity, including for 8 'negative' affect states (Worry, Sadness, Stress, Anger, Boredom, Loneliness, Depression, Anxiety) and two 'positive' ones (Enjoyment, Happiness). In addition to these individual measures, we also consider a composite measure of affective wellbeing that ranges from -8 to 2 (computed at the individual level by subtracting sum of negative affect scores from sum of positive affect scores).

While COVID-specific survey data was collected starting
March 13, 2020, and is currently available to us through August 30, 2020, the vaccination question* that is of prime interest in this report was started on July 20, 2020 (nationwide, $N=16,162$ individuals were surveyed between July 20 and August 30, 2020). To ensure that the data forms a reasonable basis for the study at this time, we computed the association between per-capita vaccination doses administered (using the most recent data available at the state level; see Materials and Methods) and the vaccine hesitancy ratio, also at the state level. Using either the Spearman's rank correlation $(r=-0.28)$ or the Pearson's correlation $(r=-0.22)$, results show the two are negatively associated $(p<0.01)$, despite vaccine doses not being uniformly available to all segments of the population.

\section{Results}

Table 1 summarizes the key results, while Fig. 1 illustrates county-level associations (horizontal axis) between vaccine hesitancy and a particular affect state (vertical axis). The first row (Pearson's correlation, without controls, between vaccine hesitancy and the affective wellbeing measure associated with a column) shows that, with high significance, vaccine hesitancy is positively correlated with all measures of positive (and composite) wellbeing except anger. Similarly, it is negatively correlated with measures of negative affective wellbeing. However, the correlation with anger is weaker by an order of magnitude compared to many of the other measures, and is only moderately significant $(<0.01<p<0.05)$. We find that it is also not robust: depending on the control in place, the result becomes insignificant, or reverses sign when it does not. Nevertheless, anger can disproportionately impact certain counties, as we describe shortly.

Other variables show greater robustness, significance and consistency. Several interesting associations emerge when we consider the controlled correlation results. White individuals have an almost 9 percent positive correlation between the composite measure of wellbeing and vaccine hesitancy. Individuals whose households fall into the middle-income bracket of $\$ 36,000-90,000$, as well as those that are employed full time, have correlations higher than $10 \%(p<0.01)$. Positive correlations are observed both among Democrats and Republicans, but the latter are more strongly correlated by 2 percent.

* If an FDA-approved vaccine to prevent coronavirus/COVID-19 was available right now at no cost, would you agree to be vaccinated? Yes/No, with No indicating vaccine hesitancy.

M.K. conceived the idea and supervised the research, and K.S. was responsible for the experimental study, background and analysis. Both contributed equally to writing.

The authors have no competing interests to declare.

${ }^{1}$ M.K. (Mayank Kejriwal) contributed equally to this work with K.S (Ke Shen).

${ }^{2}$ Correspondence should be addressed to M.K. E-mail: kejriwal@isi.edu 
Table 1. The absolute (i.e., uncontrolled) and partial correlation (Corr.) between vaccine hesitancy and individual affect states (including a composite measure), with controls including categorical variables like race, party, gender, (bracketed) income and employment status.

\begin{tabular}{|c|c|c|c|c|c|c|c|c|c|c|c|}
\hline & Enjoyment & Worry & Sadness & Stress & Anger & Happiness & Boredom & Loneliness & Depression & Anxiety & Composite \\
\hline Absolute Corr. & $0.06^{* * *}$ & -0.09 *** & $-0.06^{* * *}$ & $-0.03^{* * *}$ & $0.003^{* *}$ & $0.05^{* * *}$ & $-0.04^{* * *}$ & $-0.03^{* * *}$ & $-0.04^{* * *}$ & $-0.07^{* * *}$ & $0.08^{* * *}$ \\
\hline \multicolumn{12}{|l|}{ Partial Corr. } \\
\hline \multicolumn{12}{|l|}{ Race } \\
\hline Hispanic & 0.06 * & -0.03 & -0.04 & 0.02 & 0.04 & 0.09 * & -0.06 * & -0.01 & -0.01 & -0.04 & 0.04 \\
\hline Black & -0.01 & -0.01 & -0.04 & 0.00 & -0.05 & 0.00 & -0.02 & -0.01 & -0.03 & -0.03 & 0.03 \\
\hline Asian & 0.09 & 0.00 & 0.01 & 0.09 & 0.00 & 0.07 & -0.03 & 0.00 & 0.00 & 0.02 & 0.02 \\
\hline White & $0.06^{* * *}$ & $-0.10^{* * *}$ & $-0.06^{* * *}$ & $-0.04^{* * *}$ & 0.00 & $0.05^{* * *}$ & $-0.04 * * *$ & $-0.04 * * *$ & $-0.04 * * *$ & $-0.08^{* * *}$ & $0.09 * * *$ \\
\hline Other & 0.04 & $-0.18^{* *}$ & -0.14 & -0.17 & -0.06 & 0.12 & -0.04 & -0.02 & -0.10 & -0.09 & $0.15^{* *}$ \\
\hline \multicolumn{12}{|l|}{ Political Party } \\
\hline Neither & $0.23^{* *}$ & 0.09 & 0.04 & 0.09 & -0.07 & $0.21 *$ & 0.00 & 0.13 & 0.14 & -0.15 & 0.03 \\
\hline Democrat & $0.05^{* * *}$ & $-0.08^{* * *}$ & $-0.06^{* * *}$ & $-0.03^{* * *}$ & 0.01 & $0.05^{* * *}$ & $-0.04^{* * *}$ & $-0.03^{* * *}$ & $-0.03^{* * *}$ & $-0.06^{* * *}$ & $0.07^{* * *}$ \\
\hline Republican & $0.06^{* * *}$ & $-0.10^{* * *}$ & $-0.05^{* * *}$ & $-0.04^{* * *}$ & 0.00 & $0.06^{* * *}$ & $-0.03^{* * *}$ & $-0.04^{* * *}$ & $-0.05^{\star * *}$ & $-0.09^{* * *}$ & $0.09^{* * *}$ \\
\hline \multicolumn{12}{|l|}{ Gender } \\
\hline Male & $0.05^{* * *}$ & $-0.09 * * *$ & $-0.06^{* * *}$ & -0.02 ** & 0.02 & $0.03^{* * *}$ & $-0.05^{* * *}$ & $-0.03^{* * *}$ & $-0.04^{* * *}$ & $-0.07^{* * *}$ & $0.07^{* * *}$ \\
\hline Female & $0.08^{* * *}$ & $-0.11^{* * *}$ & $-0.07^{* * *}$ & $-0.06^{* * *}$ & -0.01 & $0.08^{* * *}$ & $-0.03^{* * *}$ & $-0.04 * * *$ & $-0.05^{* * *}$ & $-0.10^{* * *}$ & $0.10^{* * *}$ \\
\hline \multicolumn{12}{|l|}{ Income } \\
\hline Less than $\$ 36,000$ & 0.03 & $-0.09 * * *$ & -0.03 & -0.03 & 0.04 * & 0.04 ** & $-0.05^{* *}$ & $-0.06^{* \star *}$ & $-0.04 * *$ & $-0.06^{* * *}$ & $0.06^{* * *}$ \\
\hline$\$ 36,000$ to $\$ 90,000$ & $0.08^{* * *}$ & $-0.11^{\star * *}$ & $-0.08^{* * *}$ & $-0.05^{* * *}$ & $-0.03^{* *}$ & $0.07^{* * *}$ & $-0.04^{* * *}$ & $-0.06^{* * *}$ & $-0.06^{* * *}$ & $-0.09 * * *$ & $0.11^{* * *}$ \\
\hline More than $\$ 90,000$ & $0.06^{* * *}$ & $-0.08^{* * *}$ & $-0.05^{* * *}$ & -0.02 & 0.01 & $0.05^{* * *}$ & $-0.03^{* * *}$ & $-0.02 * *$ & $-0.03^{* * *}$ & $-0.06^{* * *}$ & $0.07^{* * *}$ \\
\hline \multicolumn{12}{|l|}{ Employment } \\
\hline Full-time & $0.08^{* * *}$ & $-0.13^{* * *}$ & $-0.08^{* * *}$ & $-0.09 * * *$ & -0.01 & $0.07^{* * *}$ & -0.02 * & $-0.04^{* * *}$ & $-0.06^{* * *}$ & $-0.11^{* * *}$ & $0.12^{* * *}$ \\
\hline Part-time & $0.08^{* * *}$ & $-0.11^{* * *}$ & $-0.09 * * *$ & $-0.05^{*}$ & -0.01 & $0.08^{* * *}$ & $-0.06^{* *}$ & -0.03 & $-0.05^{*}$ & $-0.10^{* * *}$ & $0.11^{* * *}$ \\
\hline Involuntary unemp. & 0.08 ** & $-0.09 * *$ & -0.06 & $-0.10^{* *}$ & -0.01 & $0.09 * *$ & -0.02 & $-0.08 * *$ & -0.07 * & $-0.10 * *$ & $0.11^{* * *}$ \\
\hline Not in labor force & $0.04^{* * *}$ & $-0.06^{* * *}$ & $-0.02^{* *}$ & -0.01 & 0.02 * & $0.04^{* * *}$ & $-0.04^{* * *}$ & $-0.02 *$ & -0.01 & $-0.04^{* * *}$ & $0.04^{* * *}$ \\
\hline
\end{tabular}

Income is the total annual household income before taxes. Involuntary unemployed (unemp.) are those are unemployed but actively looking for job, Not in labor force includes full-time students, homemakers, retirees and those who are unemployed and not looking for work. *, **, *** respectively indicate $p<0.1, p<0.05$ and $p<0.001$ compared to 0 .

In Fig. 1, each association was quantified by computing the absolute correlation (similar to the first row in Table 1) but for each county, rather than nationwide. We also illustrate the degree of political affiliation (of survey respondents) in that county by appropriately shading in the circle. Participant political affiliation was measured using a fairly established question, which we state for completeness in Materials and Methods. The results are consistent with Table 1; we do not see clear divisions among party lines.

\section{Discussion}

One hypothesis that is suggested by the data in Table 1 (but that we cannot evaluate in this brief report) is that individuals who were able to transition into remote work and are comfortably employed score higher on affective wellbeing (which is natural) but are also vaccine-hesitant. Complacency may be a reason i.e., these individuals may feel that they can afford to wait and evaluate the efficacy of the vaccine since they may be able to avail of the opportunity to keep working from home in the near-future. If true, employer-based incentives to vaccinate may have some promise.

Despite what some news articles mention about minorities and military personnel being vaccine-hesitant (which the evidence herein does not necessarily refute) (1), the correlation between being Black, Hispanic or Asian, and composite wellbeing is much lower than for White individuals. Correlations are also lower for individuals who belong to households with income below $\$ 36,000$, where minority communities are known to be disproportionately represented. These nuances suggest that statements in the popular press about minorities and vaccine hesitancy deserve further qualification. Particularly, individuals in the poorest households have the least correlation between positive wellbeing and vaccine hesitancy.

In Fig. 1, among some of the negative affect states (including worry and stress), there is considerable dispersion compared to the positive affect states. Perhaps the most alarming observation is the set of outlier counties that have high positive correlations between vaccine hesitancy and negative affect states like stress and anger. If the trend holds in these counties when vaccines are rolled out to the larger population, vulnerable populations (that are already experiencing negative wellbeing) may be most at risk of not receiving vaccination.

A brief investigation of some counties revealed that, among the top 5 counties with the highest anger, 3 of the 5 counties have positive and significant association between anger and vaccine hesitancy, and 3 of 5 counties also have vaccine hesitancy ratios higher than 0.3. Again, there is no clear separation by political affiliation (three of the counties are highly Democrat, and the other two are Republican, but all show high political partisanship), suggesting that political causal drivers, if any, are complex. More recent numbers, once available, may allow us to do county-level comparisons before and after the November elections to understand if change in administration had an influence. 


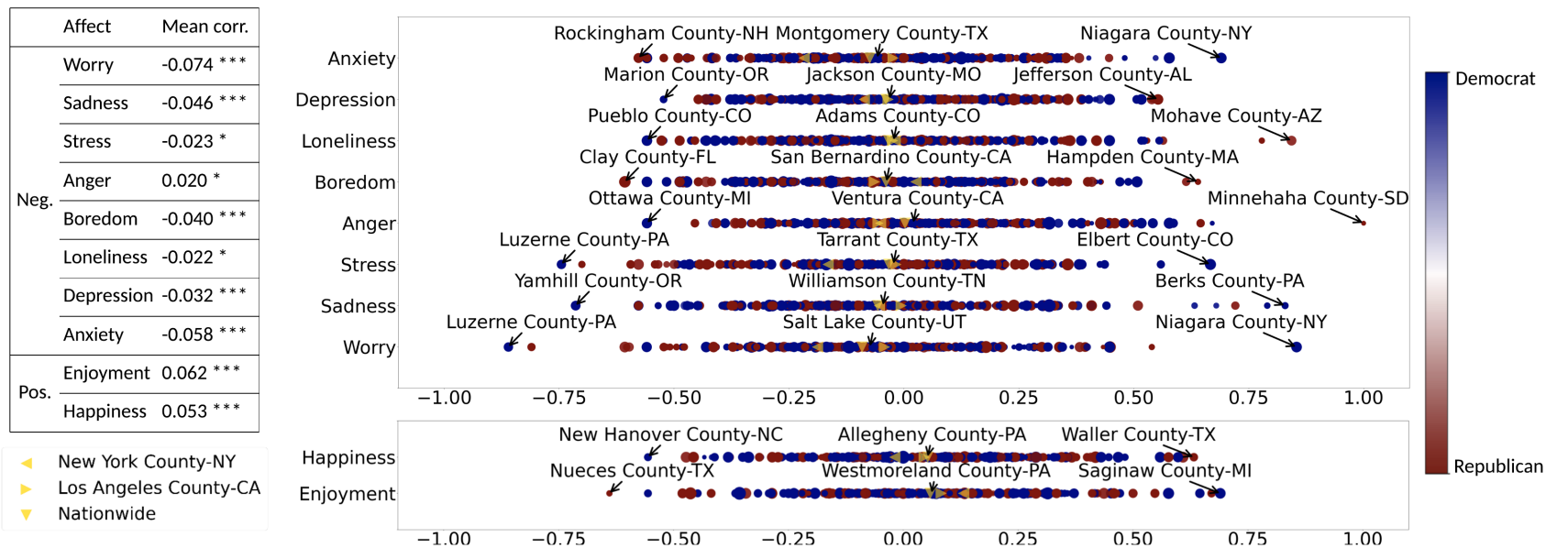

Fig. 1. A visual summary of county-level correlations between vaccine hesitancy and affect states. Smaller circles indicate less absolute vaccine hesitancy. The table on the left is the mean correlation where the mean is computed over the counties $(N=324)$.

\section{Materials and Methods}

Gallup began fielding on March 13, 2020 by launching a specific survey that collected people's responses during the COVID-19 pandemic, polling daily random samples of the Gallup Panel (a probability-based, nationally representative panel of U.S. adults). As in other Gallup surveys (such as the Gallup World Poll), questions measured aspects of subjective wellbeing (including affective wellbeing, which was the primary focus herein, and also life satisfaction), employment, health, and demographics, among others.

To measure affective wellbeing, the following was asked Did you experience the following feelings during a lot of the day yesterday with a Yes/No option for each of the following choices: Enjoyment, Worry, Sadness, Stress, Anger, Happiness, Boredom, Loneliness, Depression, Anxiety. This question is an individual-level affective wellbeing measurement, and the proportion of Yes responses for a feeling within a spatial unit (such as a county) is taken as the measure of affective wellbeing for that spatial unit. We treat Happiness and Enjoyment as positive affect states and the others as negative affect states. The composite affective wellbeing score sums the scores for an individual on each measure, and ranges from -8 (with a Yes response on all eight negative affect choices and a $N o$ response on the two positive ones), to 2 .

The vaccine hesitancy question was asked starting from July 20 , 2020: If an FDA-approved vaccine to prevent coronavirus/COVID19 was available right now at no cost, would you agree to be vaccinated? with possible response of Yes or No to investigate a person's willingness to be vaccinated. The proportion of $N o$ responses in a specific area is defined in this work as vaccine hesitancy.

Since the vaccine hesitancy question was added on July 20, the responses of interest in this study are from July 20 to August 30, 2020. Across this time period, 16,162 participants (46 percent female) from 1,936 counties responded to the survey. Other relevant statistics are provided in the SI Appendix.

For verifying the association between vaccine hesitancy and actual vaccination, we used the vaccination rate data provided by Our World in Data ${ }^{\dagger}$, which is updated daily and includes data on confirmed cases, deaths, hospitalizations, testing, and vaccinations as well as other variables of potential interest. We use the latest version (posted on February 18) that was available at the time of writing.

A participant's political affiliation is measured by asking the question In politics, as of today, with which political party do you most closely affiliate? There are four possible responses: Republican, Democrat, Independent, and Other Party. In Table 1, Neither refers to the last two options. In Fig. 1, at the county level, we assign scores of -1,1 and 0 to Democrat, Republican and Neither and get an average score for the county to measure the intensity of political affiliation.

Note that the Yes response for an affective wellbeing question is encoded as 1 (No response is encoded as 0 ), but the Yes response for the vaccination question is encoded as 0 ( $N o$ response is encoded as 1 ). The reason, of course, is that No indicates vaccine hesitancy In the county-level analysis in Fig. 1, we only retain counties that have more than 10 responses. More than 50 percent of these 324 counties show a significant (Pearson's) correlation between at least one of affect variable and vaccine hesitancy at the $p<0.1$ significance level. At the state level, 80 percent of states have significant correlations between at least one affect state and vaccine hesitancy at the $p<0.1$ level. Nationally (as indicated in the first row of Table 1), the association between individuals' affective wellbeing and vaccine hesitancy is highly significant $(p<0.01)$.

1. D Leonhardt, Underselling the vaccine (https://www.nytimes.com/2021/01/18/briefing donald-trump-pardon-phil-spector-coronavirus-deaths.html?campaign id=9\&emc=edit nn 20210218\&instance_id $=27279 \&$ nl=the- morning\&regi_id $=123188145 \&$ segment_id $=51953 \&$ te=1\&user_id=23aac8450540867b8efed81b8a5a05fb) (year?) Accessed: 2021-02-19.

2. AA Dror, et al., Vaccine hesitancy: the next challenge in the fight against covid-19. Eur. journal epidemiology 35, 775-779 (2020).

3. KO Kwok, et al., Influenza vaccine uptake, covid-19 vaccination intention and vaccine hesitancy among nurses: A survey. Int. journal nursing studies 114, 103854 (2021).

4. DA Salmon, MZ Dudley, JM Glanz, SB Omer, Vaccine hesitancy: causes, consequences, and a call to action. Vaccine 33, D66-D71 (2015).

5. SC Quinn, AM Jamison, J An, GR Hancock, VS Freimuth, Measuring vaccine hesitancy, confidence, trust and flu vaccine uptake: Results of a national survey of white and african american adults. Vaccine 37, 1168-1173 (2019).

6. P Paterson, et al., Vaccine hesitancy and healthcare providers. Vaccine 34, 6700-6706 (2016).

7. E Diener, Subjective well-being. The science well-being, 11-58 (2009).

8. E Diener, Assessing subjective well-being: Progress and opportunities. Assess. well-being, 25-65 (2009).

9. E Diener, S Oishi, L Tay, Advances in subjective well-being research. Nat. Hum. Behav. 2 253-260 (2018).

10. E Diener, RA Emmons, RJ Larsen, S Griffin, The satisfaction with life scale. J. personality assessment 49, 71-75 (1985).

11. M Joshanloo, Longitudinal associations between subjective and psychological well-being in japan: A four-year cross-lagged panel study. Pers. Individ. Differ. 134, 289-292 (2018).

12. MT Davern, RA Cummins, MA Stokes, Subjective wellbeing as an affective-cognitive construct. J. Happiness Stud. 8, 429-449 (2007)

13. IC Galinha, JL Pais-Ribeiro, Cognitive, affective and contextual predictors of subjective wellbeing. Int. J. Wellbeing 2 (2012). 